\title{
Discrimination learning in the South American tapir (Tapirella)
}

\author{
GARVIN MCCAIN, University of Texas at Arlington, Texas \\ 76010
}

Strong position responses appear related to a high level of emotional arousal. Performance abruptly improved from a chance level after a very extended period.

Over the past several years we have made a number of attempts to work with animals in zoo settings. The object of these studies has been to find means of working with animals in a zoo setting, obtain some knowledge of adequate reinforcers, identify stimuli suitable for the capacities of particular animals, and obtain some information on discrimination learning. The choice of tapirs in the present study was related to earlier use of black rhinoceroses in a series of discrimination studies (McCain \& Stepter, 1968). Since rhinos and tapirs are important members of a rather limited order, (Perissodactyla, three-toed), at least gross comparisons seem useful.

In the odd-toed order the rhinos and tapirs have a common origin and it seems likely the tapir is the more ancient form. Tapirs have been termed "living fossils" since their modern form is very close to that of the fossil remains from the Miocene period (about 12 million B.C.). Earlier but somewhat smaller forms go back at least 50 million years. Geographic distribution of tapirs is somewhat peculiar in that they are found only in Malaya, the East Indies, and Central and South America. The particular species used in this study ( $T$. terrestris) are semiaquatic and although smaller than other tapirs are still the heaviest of wild animals native to South America. They are extremely solid and very strong for their size.

\section{Subjects}

\section{METHOD}

The Ss were a male and a female, approximately four to six years old. Both Ss were captured as young animals and had spent the majority of their lives in the zoo.

Apparatus

The study was run in the Ss' normal pen, which was approximately $40 \mathrm{ft} \times 60 \mathrm{ft}$, with a stone house at the back of
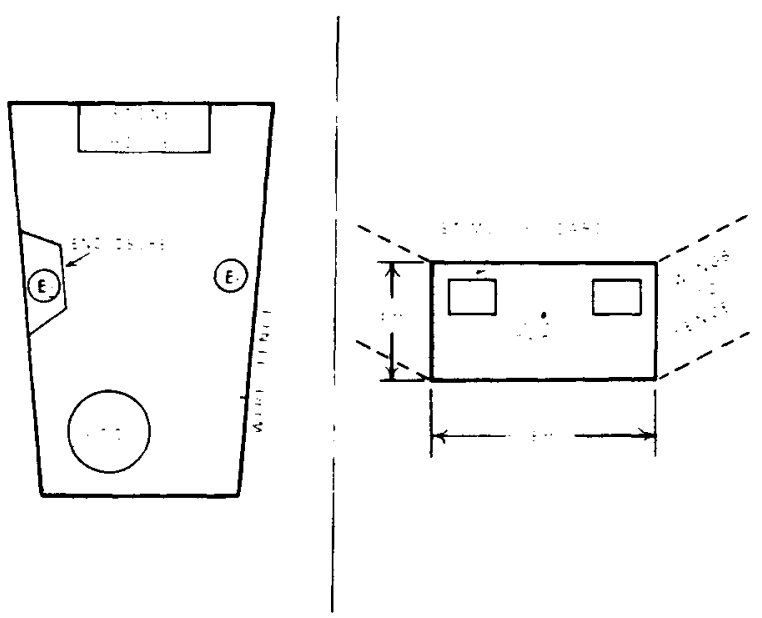

Fig. 1a (left). Sketch of tapir pen showing position of Es during discrimination problem.

Fig. 1b (right). Front view of enclosure. the pen. Figure 1-a is a sketch of the pen which also shows the apparatus and Es as they were positioned in the pen.

Figure 1-b is a sketch of the apparatus itself seen from the point of view of the tapir. The apparatus consisted of an enclosure formed by three plywood sides and a fence. The dimensions of the enclosure are shown on the sketch. The enclosure remained in the pen throughout the study. The stimulus cards were 18-in. x 18-in. plywood squares, one black and the other white. The enclosure was painted medium gray. Procedure

The Es spent a total of about $8 \mathrm{~h}$ allowing the Ss to become accustomed to their presence and trying several different food rewards. As with rhinos, the tapirs accepted a wide variety of vegetable foods plus candy and white bread. White bread was selected as a reward because of cost andease of handling. Each $S$ was trained to respond to its name while in the pen described above. In the beginning the Es stood about $6 \mathrm{ft}$ apart, while one would call the S's name and offer half a slice of bread. Gradually the distance between Es was increased and the bread was concealed until the $S$ responded. Next, one $E$ stood in the enclosure, the other outside and across the pen, as shown in Fig. 1-a. After Ss began to approach the enclosure regularly when called, the $E$ in the enclosure positioned himself behind the black card and called S. The black card was shifted from side to side on a schedule taken from the Gellerman (1939) orders. After approximately 60 trials E concealed himself in the center of the plywood enclosure and called $S$; if $S$ responded to the correct card, reward of $1 / 2$ slice of bread was given. During acquisition the female $\mathrm{S}$ was given 154 trials, the male 168 trials, at 14 trials per day.

$$
\text { RESULTS AND DISCUSSION }
$$

Working with a new species does have some possible values other than producing a learning curve. Techniques were developed to cope with these Ss. While the procedure sounds rather straightforward, the actual operation was more poorly controlled than even the usual rat study.

The results in terms of the percentage of correct responses for each $\mathrm{S}$ are shown in Fig. 2. As may be noted, both Ss had a relatively long period of responses at approximately a chance level. This period covers 70 trials for the male $S$ and 98 trials for the female $S$. The figure does not tell most of the story. Both Ss started the problem with a very strong position response. Happily, one responded left, the other right. The male $S$ broke the position response after 70 trials and, as may be seen in the graph, improvement in performance was extremely rapid from this point. The situation with the female $\mathrm{S}$ was even more extreme; she made 98 successive left responses. At this point she was given one forced trial to the right side. Her change of behavior was sudden; she made only five errors in the succeeding 56 trials. While learning in this case seems to proceed very rapidly, a description of the actual events may be useful. During the long period of position responding the Ss gave some signs that they. too. were impatient with the situation. On trials in which the white card was on their response side considerable emotional behavior was observed. The Ss would refuse to respond or make responses to the position preference without slowing down long enough for the reward to be given. Other behaviors such as crying, defecating. biting $E$, chasing leaves and running away from the experimental area seemed to indicate some sort of emotional arousal. It appears likely that the emotional arousal interfered with response flexibility.

There is at least one other point to be made. A serious 


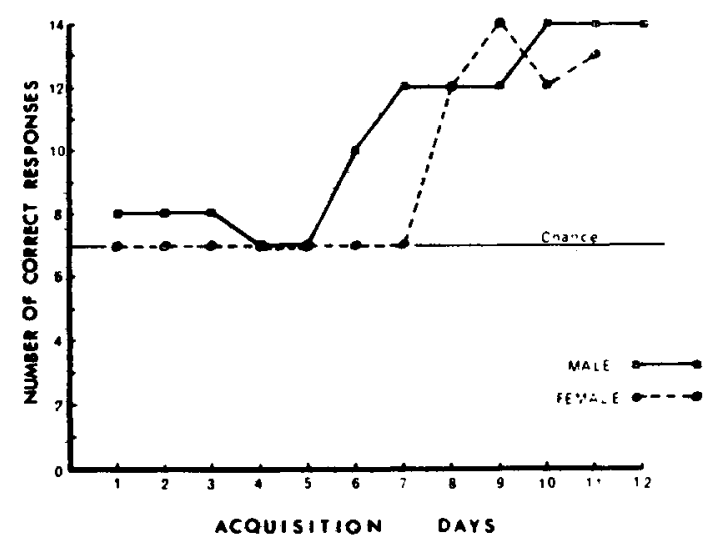

Fig. 2. Number of correct responses by days, on black-white discrimination problem.

mistake appears to have been made when it was assumed that the pretraining had effectively transferred to the acquisition situation. Note that during the first stage of pretraining $E$ plus calling the S's name became very effective stimuli which elicited a reliable approach response. During a latter stage when $E$ stood behind the stimulus there was apparently little or no association between the stimulus card and reward, in spite of the fact that reward was given about $6 \mathrm{in}$. in front of the stimulus card. It appears that $E$ had become such a potent stimulus that a later added stimulus had little or no effect. Stimuli impinging upon the receptors may or may not acquire any ability to evoke a response when another stimulus has been previously established as the critical stimulus.

As in the earlier studies on rhinos, the tendency to fixate on a particular position response was quite evident. Previous experience with the rhinos has indicated the presence of highly emotional behavior in a simple discrimination problem. In a series of studies with rhinos the tendency to give a position response decreased over problems.

\section{REFERENCES}

GELLERMAN, L. W. Chance orders of alternating stimuli in visual discrimination experiments. Journal of Genetic Psy chology, 1933, 42, 356-360.

McCAIN, G. Learning in the Black Rhinoceros (Diceros bicornis). Paper presented at American Psychological Association Convention, September, 1963.

MCCAIN, G., \& STEPTER, G. Discrimination learning and extinction in the Black Rhinoceros (Diceros bicornis). Psychonomic Science, in press.

NOTE

1. This study was made possible through the generous cooperation of the staff of the Ft. Worth Zoo. Lawrence Curtis, formerly with the Ft. Worth Zoo, was particularly helpful. Thanks are due Terry Hawles and Brian McCain who ran the Ss. Some of these data were presented at the Southwestern Psychological Association Convention, 1968. 